\title{
Protective effect of diethylcarbamazine inhibits NF-кB activation in isoproterenol-induced acute myocardial infarction rat model through the PARP pathway
}

\author{
GUOWEI JIA, MING ZAO and XIAOYU LIU \\ Department of Cardiovascular Internal Three, Cangzhou Central Hospital, Cangzhou, Hebei 061000, P.R. China \\ Received February 9, 2016; Accepted February 10, 2017
}

DOI: $10.3892 / \mathrm{mmr} .2017 .6695$

\begin{abstract}
The present study investigated the protective effect of diethylcarbamazine in inhibiting nuclear factor $(\mathrm{NF})-\kappa \mathrm{B}$ activation in isoproterenol-induced acute myocardial infarction (AMI) rats through the poly ADP ribose polymerase (PARP) pathway. Male albino Wistar rats were injected subcutaneously with isoproterenol (100 mg/kg/day) for 2 days to induce an AMI model. Diethylcarbamazine $(50 \mathrm{mg} / \mathrm{kg}$ ) was administered by gavage for 12 days prior to the isoproterenol-induced AMI. It was noted that diethylcarbamazine significantly inhibited AMI-induced casein kinase and lactate dehydrogenase levels, and reduced the AMI-induced wet heart weight to body weight ratio in AMI rats. Diethylcarbamazine treatment significantly weakened reactive oxygen species production and reduced the levels of tumor necrosis factor (TNF)- $\alpha$, interleukin- 6 and $\mathrm{NF}-\kappa \mathrm{B} / \mathrm{p} 65$ in AMI rats. Western blotting demonstrated that diethylcarbamazine significantly suppressed the AMI-induced inducible nitric oxide synthase (iNOS), transforming growth factor (TGF)- $\beta 1$, cyclooxygenase- 2 (COX-2) and PARP protein expression in AMI rats. The results demonstrated that the protective effect of diethylcarbamazine inhibited isoproterenol-induced AMI through the suppression of inflammation, iNOS, TGF- $\beta 1$, COX-2 and the PARP pathway, and revealed the clinical potential of diethylcarbamazine for therapeutic and clinical applications.
\end{abstract}

\section{Introduction}

Myocardial infarction (MI) is a major cause of cardiovascular disease, mortality and disability (1). In China, millions die of acute MI (AMI), which is regarded as a major threat to human health (2). With the extensive employment of thrombolysis and cardiac intervention therapy, in addition to the rapid advancements

Correspondence to: Mr. Guowei Jia, Department of Cardiovascular Internal Three, Cangzhou Central Hospital, 201 Inhua Road, Cangzhou, Hebei 061000, P.R. China

E-mail: guowei.jia111@163.com

Key words: diethylcarbamazine, acute myocardial infarction, inducible nitric oxide synthase, transforming growth factor- $\beta 1$, cyclooxygenase-2, poly ADP ribose polymerase in drug therapy for treating AMI, myocardium damage in patients with AMI has been greatly ameliorated and the prognosis markedly improved (3). However, there remain patients who cannot be aided with timely revascularization and so suffer irreversible death of the myocardium and ventricular reconstruction (4).

Ventricular reconstruction refers to the changes in the morphological structures of myocardial cells and the intercellular substance caused by the activation of neurohumoral regulatory mechanisms, inflammation and cytokines (5). Cardiac structures and functions become altered according to certain patterns (6). The progressive enlargement and changes in appearance of the ventriculus sinister include changes in ventricular volume, shape, ventricular wall thickness and cardiac structures, which lead to abnormalities of cardiac structure and hemodynamics, progressive dilation of the left ventricle and the decrease of systolic functions (7). Finally, heart failure and mortality may occur (8).

Poly ADP-ribose polymerase (PARP) is widely expressed in eukaryotic cells. Activated PARP is involved in DNA repair (9). Ischemia and hypoxia result in DNA damage and the excessive activation of PARP, can cause the exhaustion of ATP resources (10). As a result of such dysfunction, the cells die. The activities of PARP can be inhibited by 3-aminobenzamide, which can also reduce the consumption of ATP during the process of DNA repair (11). It has been employed for studying multiple tissue ischemic injuries and possesses potential for ischemic myocardium treatment (9).

Previous studies have identified that diethylcarbamazine can be used to inhibit vasoconstriction, reduce systemic arterial pressures and block hypoxic pulmonary vasoconstriction (12). As a diethylcarbamazine, hetrazan has been reported to inhibit the contraction of isolated vascular circles, decrease systemic arterial and pulmonary arterial pressure (13). Trials on animal models have suggested that intravenous injection of diethylcarbamazine can temporarily change cardiac functions and decrease the heart rate (14). The aim of the present study was to investigate the protective effect of diethylcarbamazine against isoproterenol-induced AMI in a rat model and investigate a possible mechanism for its protective effect.

\section{Materials and methods}

Animals, induction of AMI and experimental protocol. The present study was performed in accordance with the Guide 
for the Care and Use of Laboratory Animals of Cangzhou Central Hospital. Male albino Wistar rats $(\mathrm{n}=24 ; 230-250 \mathrm{~g})$ were obtained from the Animal Experimental Center of Hebei Medical University (Shijiazhuang, China) and housed in standard polypropylene cages under a 12:12 h light:dark cycle at a constant temperature of $23 \pm 2^{\circ} \mathrm{C}$ and an ambient humidity of $55 \pm 5 \%$. Isoproterenol (Sigma-Aldrich; Merck KGaA, Darmstadt, Germany) was injected subcutaneously to rats $(100 \mathrm{mg} / \mathrm{kg} / \mathrm{day})$ to create the AMI model. All the rats were randomly divided into four groups consisting of 6 rats each: Sham, diethylcarbamazine, AMI model and AMI model + diethylcarbamazine group. In the sham and AMI model groups, normal rats and isoproterenol-induced AMI rats were injected with distilled water. In the diethylcarbamazine and the AMI model + diethylcarbamazine groups, diethylcarbamazine $(50 \mathrm{mg} / \mathrm{kg} /$ day $)$ was administered to the rats by gavage for 12 days, prior to induction of the AMI with isoproterenol.

Tissue weights and histopathological examination. The animals were euthanized with $35 \mathrm{mg} / \mathrm{kg}$ pentobarbital 2 days after induction of the AMI model, and the hearts were removed and weighed. The wet heart weight to body weight ratio was calculated to assess the degree of myocardial weight gain. Hearts samples were fixed in $10 \%$ buffered formalin prior to being embedded in paraffin wax and sectioned at $5 \mu \mathrm{m}$ thickness. Sections were stained with hematoxylin and eosin.

Measurement of casein kinase (CK), lactate dehydrogenase $(L D H)$, reactive oxygen species $(R O S)$, inflammation response and nuclear factor $(N F)-\kappa B$ activation. Serum samples were extractedfrom the venacavafollowing theisoproterenol-induced AMI model. Supernatant was collected at 5,000 x g for $10 \mathrm{~min}$ at $4^{\circ} \mathrm{C}$. The $\mathrm{CK}$ (A032, Nanjing Jiancheng Bioengineering Institute, Nanjing, China), LDH (E-EL-R0338c), tumor necrosis factor (TNF)- $\alpha$ (-EL-R0019c), interleukin (IL) -6 (E-EL-R0015c, Elabscience) and NF-кB/p65 (E-EL-R0674c) (all from Elabscience Biotechnology, Co., Ltd., Bethesda, MD, USA) activities were measured using commercial ELISA kits according to the manufacturer's protocols.

Western blot analysis. Hearts were removed and then homogenized by a Wheaton overhead stirrer. Homogenates were centrifuged at 3,000 x g for $10 \mathrm{~min}$ and the supernatant collected. Protein concentrations were determined with a bicinchoninic acid protein assay kit (Thermo Scientific Inc., Waltham, MA, USA). The proteins (40 mg) were separated with $10 \%$ sodium dodecyl sulfate-polyacrylamide by gel electrophoresis and electrophoretically transferred onto nitrocellulose membranes (Bio-Rad Laboratories, Inc., Hercules, CA, USA). Membranes were blocked with 5\% nonfat milk in TBS- $0.1 \%$ Tween-20 (TBS-T) for $1 \mathrm{~h}$ and were incubated at room temperature, for $2 \mathrm{~h}$, with anti-COX-2 (1:2,000), anti-transforming growth factor (TGF)- $\beta 1(1: 4,000)$, anti-inducible nitric oxide synthase (iNOS; 1:2,000), anti-PARP $(1: 3,000)$ and anti- $\beta$-actin $(1: 4,000)$ (all from Abcam, Cambridge, CA, USA). Following washing in TBS-T, the membranes were incubated with horseradish peroxidase-conjugated anti-rabbit secondary antibody (1:5,000; Abcam, CA, USA) for $1 \mathrm{~h}$ and $30 \mathrm{~min}$ at room temperature and visualized with an enhanced chemiluminescence reagent (Santa Cruz Biotechnology, Inc., Dallas, TX, USA). Intensity of each band was determined using the ImageJ program version 1.38 (National Institutes of Health, Bethesda, MD, USA).

Statistical analyses. Data are expressed as the mean \pm standard deviation using SPSS 17.0 (SPSS, Inc., Chicago, IL, USA). One-way analysis of variance was used to assess differences between the groups, followed by Tukey's test. $\mathrm{P}<0.05$ was considered to indicate a statistically significant difference.

\section{Results}

Protective effect of diethylcarbamazine inhibits cardiac function in isoproterenol-induced AMI rats. The protective effect of diethylcarbamazine on the cardiac function of isoproterenol-induced AMI rats was evaluated (Fig. 1). Following diethylcarbamazine treatment $(50 \mathrm{mg} / \mathrm{kg})$ for 12 days, no significant inter-group difference in cardiac functions was observed between the control and the diethylcarbamazine-alone groups $(\mathrm{P}>0.05)$. The levels of $\mathrm{CK}$ and $\mathrm{LDH}$ in the isoproterenol-induced AMI model group were higher compared with those of the control group $(\mathrm{P}<0.01)$. However, treatment with diethylcarbamazine significantly inhibited the AMI-induced CK and LDH levels in AMI rats $(\mathrm{P}<0.01)$.

Protective effect of diethylcarbamazine reduces the wet heart weight to body weight ratio in AMI rats. Following diethylcarbamazine treatment in AMI rats, the wet heart weight to body weight ratio of the control group was similar to the AMI model group. Compared with the control group, the wet heart weight to body weight ratio of the AMI model group was significantly increased $(\mathrm{P}<0.01)$. Treatment with diethylcarbamazine significantly reduced the AMI-induced wet heart weight to body weight ratio in AMI rats $(\mathrm{P}<0.01$; Fig. 2$)$.

Protective effect of diethylcarbamazine inhibits ROS production in AMI rats. To evaluate the protective effect of diethylcarbamazine on oxidative stress in AMI rats, ROS production was measured to estimate the protective effect of diethylcarbamazine on AMI (Fig. 3). No significant difference was observed between the control and diethylcarbamazine-alone groups $(\mathrm{P}>0.05)$. In the AMI model group, ROS production was significantly enhanced compared with the control group $(\mathrm{P}<0.01)$. Diethylcarbamazine treatment significantly weakened ROS production in AMI rats $(\mathrm{P}<0.01)$.

Protective effect of diethylcarbamazine inhibits inflammation response in isoproterenol-induced AMI rats. TNF- $\alpha$, IL-6 and NF- $\kappa$ B/p65 activity was detected using ELISA kits to evaluate the protective effect of diethylcarbamazine on AMI (Fig. 4). No significant difference was observed in the level of TNF- $\alpha$, IL- 6 and NF- $\kappa$ B/p65 between the control and the diethylcarbamazine-alone groups $(\mathrm{P}>0.05)$. Compared with control group, the level of TNF- $\alpha$, IL-6 and NF- $\kappa \mathrm{B} / \mathrm{p} 65$ were significantly increased in the AMI model group $(\mathrm{P}<0.01)$. However, pretreatment with diethylcarbamazine significantly reduced the AMI-induced TNF- $\alpha$, IL- 6 and NF- $\kappa$ B/p65 levels $(\mathrm{P}<0.01)$. 
A

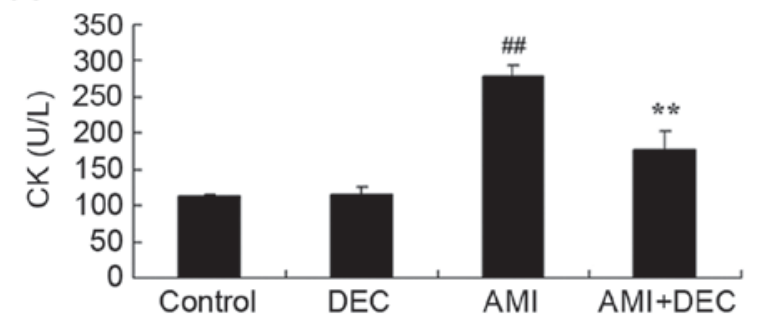

B

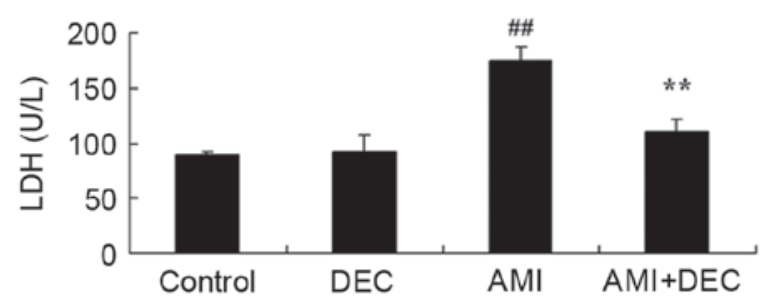

Figure 1. Protective effect of diethylcarbamazine inhibits cardiac function in isoproterenol-induced AMI rats. Diethylcarbamazine reduces (A) CK and (B) LDH in isoproterenol-induced AMI rats. ${ }^{\# \#} \mathrm{P}<0.01$ vs. control group and ${ }^{* * *} \mathrm{P}<0.01$ vs. AMI model group. CK, casein kinase; Control, control group; DEC, diethylcarbamazine-alone group; AMI, acute myocardial infarction model group; AMI + DEC, AMI model + diethylcarbamazine treated group. LDH, lactate dehydrogenase.

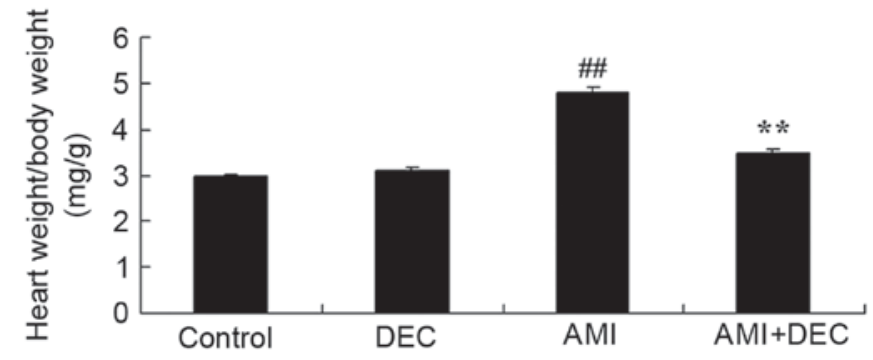

Figure 2. Diethylcarbamazine reduces infarct size in isoproterenol-induced AMI rats. ${ }^{\# \#} \mathrm{P}<0.01$ vs. control group and ${ }^{* *} \mathrm{P}<0.01$ vs. with $\mathrm{AMI}$ model group. Control, control group; DEC, diethylcarbamazine-alone group; AMI, acute myocardial infarction model group; AMI + DEC, AMI model + diethylcarbamazine treated group.

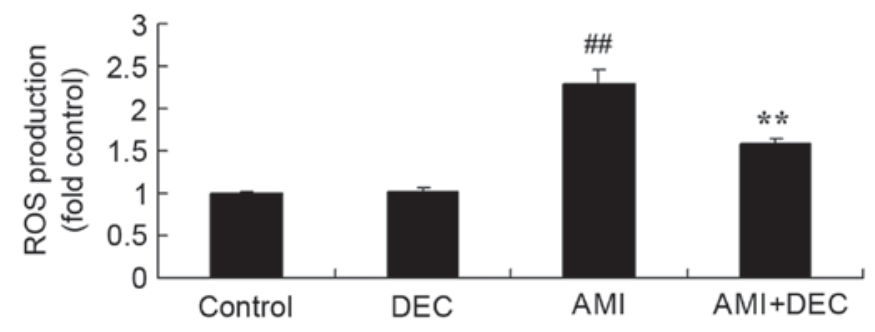

Figure 3. Protective effect of diethylcarbamazine inhibits ROS production in isoproterenol-induced AMI rats. ${ }^{\# \#} \mathrm{P}<0.01$ vs. and ${ }^{* *} \mathrm{P}<0.01$ vs. AMI model group. ROS, reactive oxygen species; Control, control group; DEC, diethylcarbamazine-alone group; AMI, acute myocardial infarction model group; AMI + DEC, AMI model + diethylcarbamazine treated group.

Protective effect of diethylcarbamazine inhibits COX-2 expression in AMI rats. No significant inter-group difference was identified between the control and the diethylcarbamazine-alone groups for COX-2 expression ( $\mathrm{P}>0.05)$. COX-2 expression in the AMI model group was higher compared with the control group $(\mathrm{P}<0.01 ;$ Fig. 5). Compared with the AMI model group, pretreatment with diethylcarbamazine noticeably reduced the AMI-induced COX-2 protein expression $(\mathrm{P}<0.01)$.

Protective effect of diethylcarbamazine reduces TGF- $\beta 1$ expression in AMI rats. No significant changes were observed in protein expression of TGF- $\beta 1$ between the control group and the diethylcarbamazine-alone group. The TGF- $\beta 1$ protein expression was notably induced by AMI, compared with the control group $(\mathrm{P}<0.01)$. Diethylcarbamazine significantly
A

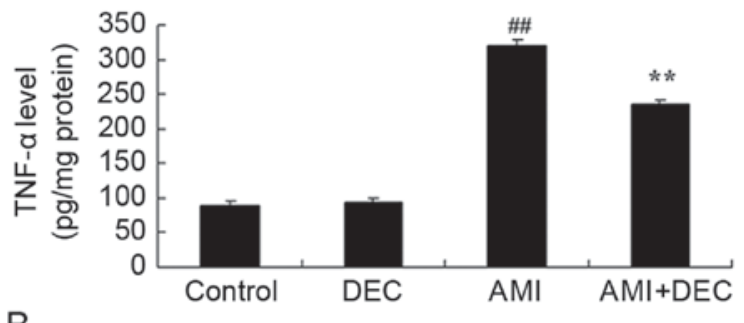

B

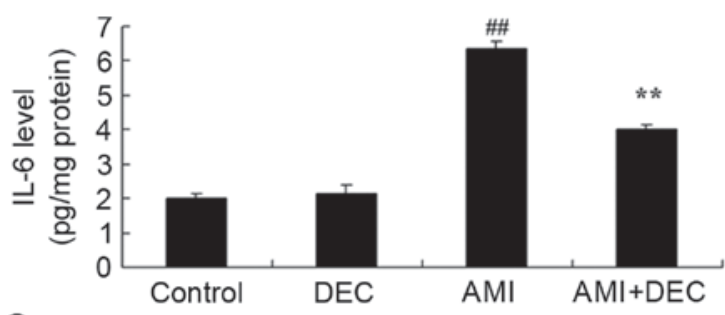

C

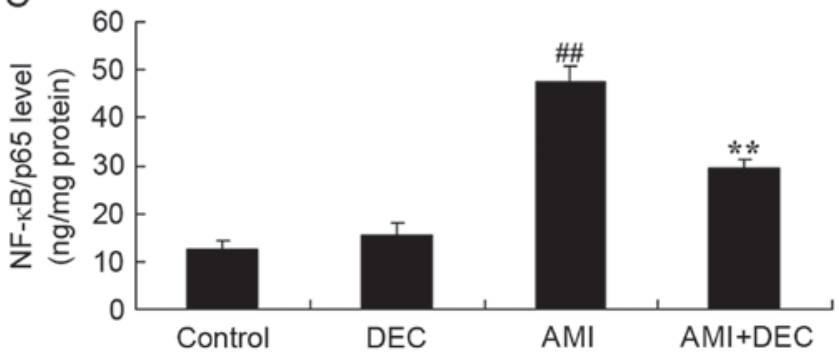

Figure 4. Protective effect of diethylcarbamazine inhibits inflammation response in isoproterenol-induced AMI rats. Protective effect of diethylcarbamazine reduced (A) TNF- $\alpha$, (B) IL-6 and (C) NF- $\kappa$ B/p65 level in isoproterenol-induced AMI rats. ${ }^{\# \#} \mathrm{P}<0.01$ vs. control group and ${ }^{* * *} \mathrm{P}<0.01$ vs. AMI model group. TNF, tumor necrosis factor; Control, control group; DEC, diethylcarbamazine-alone group; AMI, acute myocardial infarction model group; AMI + DEC, AMI model + diethylcarbamazine treated group; IL,

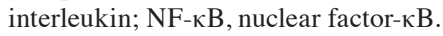

suppressed the AMI-induced TGF- $\beta 1$ protein expression $(\mathrm{P}<0.01$; Fig. 6).

Protective effect of diethylcarbamazine reduces iNOS expression in AMI rats. To explore the protective effect of diethylcarbamazine on iNOS expression in AMI rats, iNOS protein expression was measured using western blotting (Fig. 7). No significant difference was observed between the control and the diethylcarbamazine-alone groups. Compared with the control 
A

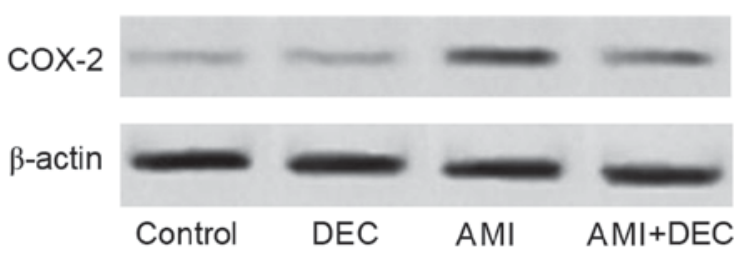

B

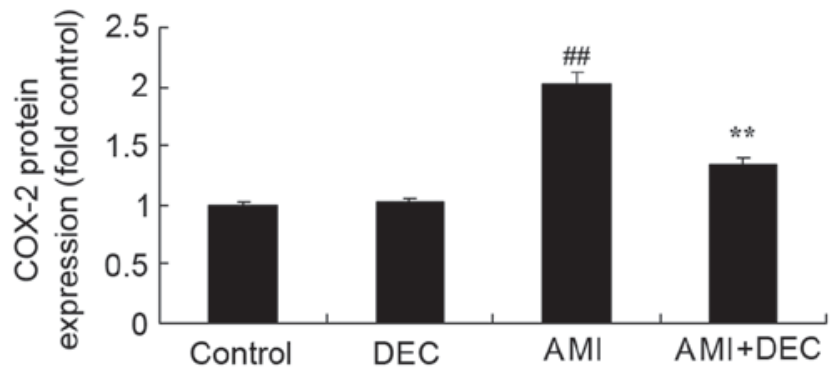

Figure 5. Diethylcarbamazine inhibits COX-2 expression in isoproterenol-induced AMI rats. (A) Protective effect of diethylcarbamazine inhibits COX-2 expression and (B) statistical analysis of COX-2 expression in isoproterenol-induced AMI rats. ${ }^{\# / P}<0.01$ vs. control group and ${ }^{* *} \mathrm{P}<0.01$ vs. AMI model group. COX-2, cyclooxygenase-2; Control, control group; DEC, diethylcarbamazine-alone group; AMI, acute myocardial infarction model group; AMI + DEC, AMI model + diethylcarbamazine treated group.

A

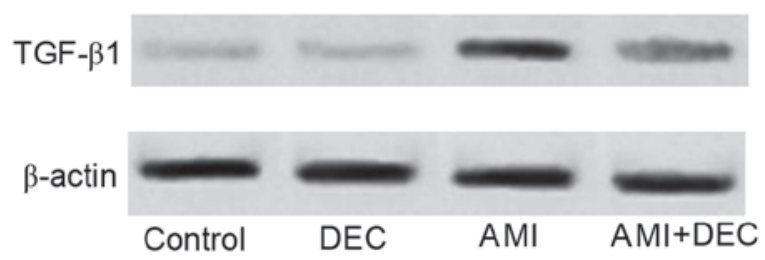

$\mathrm{B}$

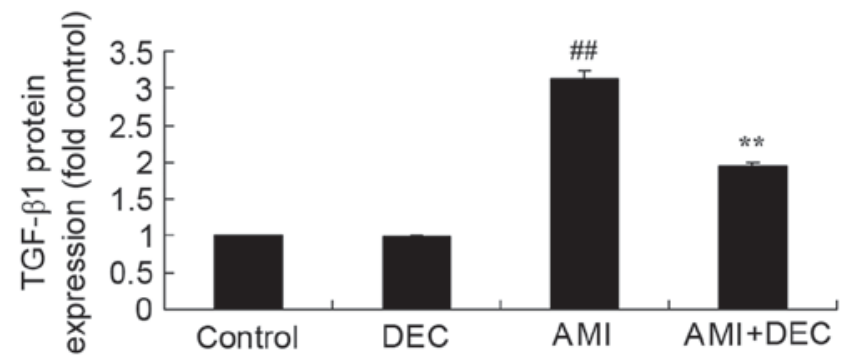

Figure 6. Diethylcarbamazine inhibits TGF- $\beta 1$ expression in isoproterenol-induced AMI rats. (A) Protective effect of diethylcarbamazine inhibits TGF- $\beta 1$ expression and (B) statistical analysis of TGF- $\beta 1$ expression in isoproterenol-induced AMI rats. ${ }^{\# \#} \mathrm{P}<0.01$ vs. control group and ${ }^{* *} \mathrm{P}<0.01$ vs. AMI model group. TGF- $\beta 1$, transforming growth factor- $\beta 1$; Control, control group; DEC, diethylcarbamazine-alone group; AMI, acute myocardial infarction model group; AMI + DEC, AMI model + diethylcarbamazine treated group.

A

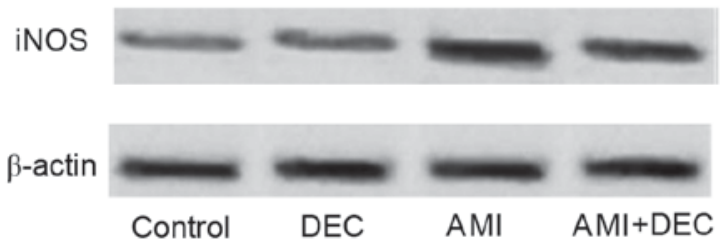

$\mathrm{B}$

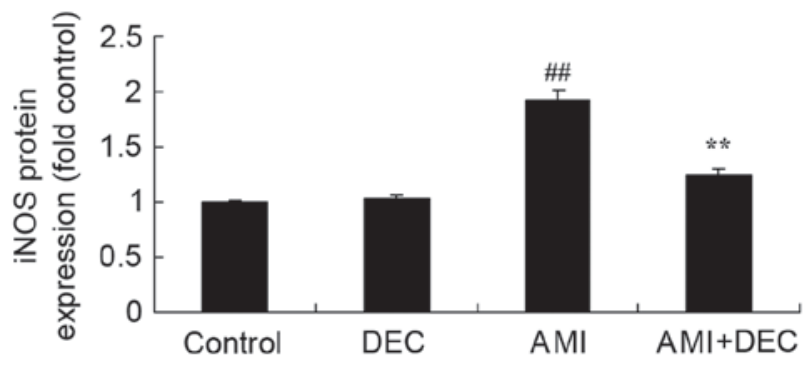

Figure 7. Protective effect of diethylcarbamazine inhibits iNOS expression in isoproterenol-induced AMI rats. (A) Protective effect of diethylcarbamazine inhibits iNOS expression and (B) statistical analysis of iNOS expression in isoproterenol-induced AMI rats. ${ }^{\# \# ~} \mathrm{P}<0.01$ vs. control group; ${ }^{* *} \mathrm{P}<0.01$ vs. AMI model group. iNOS, inducible nitric oxide synthase; Control, control group; DEC, diethylcarbamazine-alone group; AMI, acute myocardial infarction model group; AMI + DEC, AMI model + diethylcarbamazine treated group.

group, iNOS protein expression was significantly increased in AMI rats $(\mathrm{P}<0.01)$. The activation of iNOS protein expression was significantly attenuated by diethylcarbamazine in AMI rats $(\mathrm{P}<0.01)$.

Protective effect of diethylcarbamazine inhibits PARP expression in AMI rats. To further explore the protective effect of diethylcarbamazine on PARP expression in AMI rats, PARP protein expression levels were measured using western blotting (Fig. 8). No significant difference was observed between the control and diethylcarbamazine-alone groups for PARP expression $(\mathrm{P}>0.05)$. However, AMI significantly increased
PARP expression compared with the control group $(\mathrm{P}<0.01)$. Diethylcarbamazine significantly suppressed the AMI-induced PARP protein expression in AMI rats, compared with that of AMI model group $(\mathrm{P}<0.01)$.

\section{Discussion}

As a common cardiovascular disease, the morbidity of AMI is a leading health problem. With advances in medical technologies the mortality rates of AMI have decreased to a certain extent (7). However, survivors still experience complications, including recurrent AMI or refractory cardiac 
A

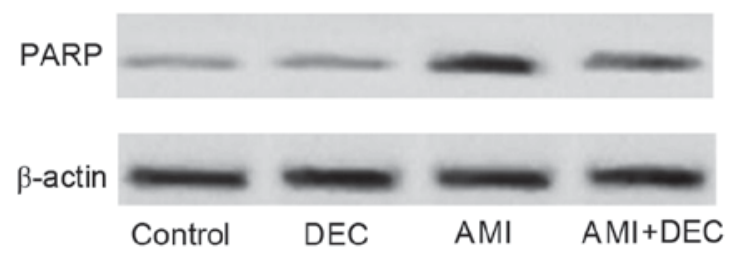

$\mathrm{B}$

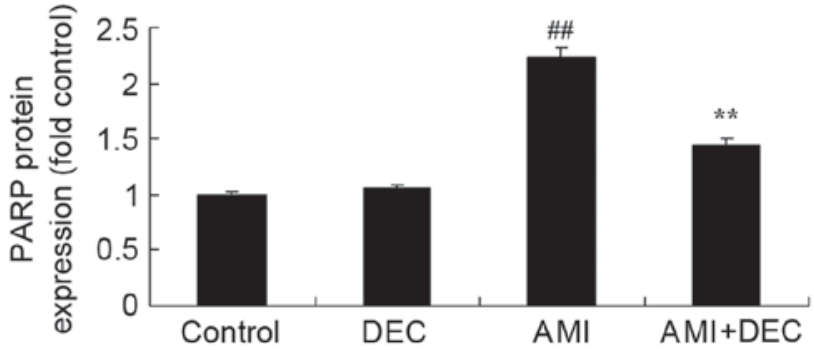

Figure 8. Protective effect of diethylcarbamazine inhibits PARP expression in isoproterenol-induced AMI rats. (A) Protective effect of diethylcarbamazine inhibits PARP expression and (B) statistical analysis of PARP expression in isoproterenol-induced AMI rats. ${ }^{\# \# P<0.01 ~ v s . ~ c o n t r o l ~ g r o u p ; ~}{ }^{* *} \mathrm{P}<0.01$ vs. AMI model group. PARP, poly ADP ribose polymerase; Control, control group; DEC, diethylcarbamazine-alone group; AMI, acute myocardial infarction model group; AMI + DEC, AMI model + diethylcarbamazine treated group.

insufficiency (15). Data have demonstrated that mortality rates of AMI and cardiac failure are $\sim 80 \%$ (15). Consequently, assessment of the myocardial systolic function of the whole and local left ventricle can accurately confirm the functional status of the ischemic myocardium; leading to an early diagnose of ischemic heart disease and useful prognosis (16). The present study investigated whether treatment with diethylcarbamazine significantly inhibited AMI-induced CK and LDH levels, and reduced the AMI-induced wet heart weight to body weight ratio in AMI rats.

An inflammatory response can be directly triggered by ROS produced by ischemic tissues (17). When the formation of ROS is much higher than the load of the endogenous antioxidant defense system, cellular damage, mediated by free radicals, occurs (18). ROS can trigger cascade reactions of inflammatory cytokines and chemotactic factors through activation of the inflammasome of myocardial cells (19). The formation of inflammasome can promote the production and activation of IL-1 which is a classic proinflammatory factor promoting the inflammatory mediator expression in infarcted myocardium (20). The present study identified that diethylcarbamazine treatment significantly weakened ROS production in AMI rats.

Inflammatory reactions have an important role in ventricular remodeling following AMI. Ventricular remodeling includes the inflammatory cascade triggered by myocyte necrosis at the infarcted zone, recruitment of inflammatory cells at the infarcted zone and upregulation of proinflammatory cytokines (21). Later, necrotic myocardial cells are replaced by collagenous fibers, resulting in the formation of glial scars, cardiomyocyte hypertrophy at the non-infarcted areas, degradation of myocardial matrix and reconstruction of matrix (22). This process involves the inflammatory responses and consequently the repair process following AMI is a complex development with related pathways mediated by inflammation as an important link (22). Continuous inflammatory responses following AMI triggers myocardial damage, fibrosis and the expansion of AMI, finally resulting in the deterioration of the cardiac function (22). A significant inhibition of TNF- $\alpha$, IL-6 and NF- $\kappa$ B/p65 levels was observed following treatment with diethylcarbamazine in AMI rats.

During the inflammatory response process, iNOS is activated and excessive nitric oxide (NO) is produced, an important factor in the death of transplanted cells (23). As an important signal transduction molecule, NO serves an essential role in physiological processes and has important effects on the progression of a number of diseases (24). In the present study, diethylcarbamazine significantly increased iNOS protein expression in AMI rats. da Silva et al (14) indicated that diethylcarbamazine prevented alcoholinduced liver injury via NF- $\mathrm{B}, \mathrm{COX}-2$ and iNOS in C57BL/6 mice.

As major effector cells of myocardial fibrosis, myofibroblasts can proliferate, synthesize and secrete a number of bioactivators, resulting in the increase of collagen deposition in mesenchyme, disproportionality and disordered arrangement, which are the pathological basis of AMI. The functions of TGF- $\beta 1$ in this process have been investigated (25). TGF- $\beta 1$ has strong chemotaxis to fibroblasts, which stimulates it to secrete a considerable amount of extracellular matrix (12). Studies have suggested that the expression of collagen I and III would increase in cardiac muscle tissues with TGF- $\beta 1(25,26)$. The present study demonstrated that diethylcarbamazine significantly suppressed the AMI-induced expression of TGF- $\beta 1$ protein. Rocha et al (27) confirmed that diethylcarbamazine reduces chronic inflammation and fibrosis in liver injury by decreasing IL- $1 \beta, \mathrm{COX}-2, \mathrm{NF}-\kappa \mathrm{B}$, interferon- $\gamma$ and TGF- $\beta$ expression.

When stimulated by hypoxia-ischemia and pro-inflammatory factors, high levels of arachidonate is released and prostaglandin (PG) H2 synthesis is catalyzed by COX-1 and COX-2. Although their catalyzation principles are similar, the catalytic action rate of COX-2 on arachidonic acid is four times that of COX-1 (28). Under the actions of modifying enzymes, PGH2 can be transformed into other PGs, including PGE2, PGI2, PGD2 and thromboxane A2. PGs exert their systemic effects via autocrine and paracrine mechanisms with signal switching conducted in the surrounding environment (29). The upregulation of COXs in myocardial cells can increase the contents of PGs in cardiac muscle tissues and can therefore has an important regulatory role in myocardial cells. A previous study indicated that COX-2 has a harmful role in ischemic myocardium and the inhibition of COX-2 can effectively protect the myocardium (30). In the present study, diethylcarbamazine significantly reduced the AMI-induced COX-2 protein expression in rats. Ribeiro et al (31) demonstrated that diethylcarbamazine attenuates carrageenan-induced lung injury in rats via COX-2 and iNOS expression. 
A key factor for necrocytosis or apoptosis is the level of ATP. If there is a store of ATP, injured cells tend to undergo apoptosis (32). If energy is depleted, necrocytosis occurs. As a selective inhibitor of PARP, 3-aminobenzamide can reduce the consumption of ATP in injured cells and transform necrotic cells to normal cells. A PARP inhibitor can significantly reduce the death of ischemic neurons and protect against injury caused by reperfusion following focal cerebral ischemia (9). The increase of PARP activation increases cell death. When ischemic damage is mild and DNA injuries are rather limited, DNA repair takes the lead and cells tolerate ischemia and survive. Following severe ischemic damage, multiple DNA damage and DNA repair mechanisms are activated; however, PARP inhibition is initiated by apoptosis signaling, which can be harmful or beneficial (11). Therefore, using an optimal dose of PARP inhibitors has a significant value in overcoming these damaged mechanisms. The present study identified that diethylcarbamazine significantly suppressed AMI-induced PARP protein expression in rats. Santos et al (13) suggested that diethylcarbamazine inhibits $N F-\kappa B$ activation via PARP in mice with acute carrageenan-induced lung injury.

In conclusion, to the best of our knowledge, the present study is the first to demonstrate that the protective effect of diethylcarbamazine inhibits $\mathrm{NF}-\kappa \mathrm{B}$ activation in isoproterenol-induced AMI rats and exerts a protective effect on AMI through the suppression of inflammation, iNOS, TGF- $\beta 1$, COX-2 and PARP, revealing the clinical potential of diethylcarbamazine for therapeutic and clinical applications.

\section{References}

1. Ritsinger V, Malmberg K, Mårtensson A, Rydén L, Wedel H and Norhammar A: Intensified insulin-based glycaemic control after myocardial infarction: Mortality during 20 year follow-up of the randomised Diabetes Mellitus Insulin Glucose Infusion in Acute Myocardial Infarction (DIGAMI 1) trial. Lancet Diabetes Endocrinol 2: 627-633, 2014

2. Suh JW, Yoon YE, Oh IY, Yoon CH, Cho YS, Youn TJ, Chae IH and Choi DJ: A single-center prospective randomized controlled trial evaluating the safety and efficacy of IntraCoronary Erythropoietin delivery BEfore Reperfusion: Gauging infarct size in patients with acute ST-segment elevation myocardial infarction. Study design and rationale of the 'ICEBERG Trial'. Contemp Clin Trials 35: 145-150, 2013.

3. Peng Y, Fu X, Li W, Geng W, Xing K, Ru L, Sun J and Zhao Y: Effect of intracoronary anisodamine and diltiazem administration during primary percutaneous coronary intervention in acute myocardial infarction. Coron Artery Dis 25: 645-652, 2014.

4. Pellaton C, Cayla G, Silvain J, Zeymer U, Cohen M, Goldstein P, Huber K, Pollack C Jr, Kerneis M, Collet JP, et al: Incidence and consequence of major bleeding in primary percutaneous intervention for ST-elevation myocardial infarction in the era of radial access: An analysis of the international randomized Acute myocardial infarction Treated with primary angioplasty and intravenous enoxaparin or unfractionated heparin to Lower ischemic and bleeding events at short- and Long-term follow-up trial. Am Heart J 170: 778-786, 2015.

5. Yamaguchi A, Adachi H, Kawahito K, Murata S and Ino T: Left ventricular reconstruction benefits patients with dilated ischemic cardiomyopathy. Ann Thorac Surg 79: 456-461, 2005.

6. Manson A, Poyade M and Rea P: A recommended workflow methodology in the creation of an educational and training application incorporating a digital reconstruction of the cerebral ventricular system and cerebrospinal fluid circulation to aid anatomical understanding. BMC Med Imaging 15: 44, 2015.

7. Hu SS, Fan HG, Zheng Z, Feng W, Wang W, Song YH, Wang LQ, Yuan X and Zhang SJ: Left ventricular reconstruction with no-patch technique: Early and late clinical outcomes. Chin Med J (Engl) 123: 3412-3416, 2010.
8. Vargas-Barron J, Antunez-Montes OY, Roldán FJ, ArandaFrausto A, González-Pacheco H, Romero-Cardenas Á and Zabalgoitia M: Myocardial rupture in acute myocardial infarction: Mechanistic explanation based on the ventricular myocardial band hypothesis. Rev Invest Clin 67: 318-322, 2015.

9. Yao L, Huang K, Huang D, Wang J, Guo H and Liao Y: Acute myocardial infarction induced increases in plasma tumor necrosis factor-alpha and interleukin-10 are associated with the activation of poly (ADP-ribose) polymerase of circulating mononuclear cell. Int J Cardiol 123: 366-368, 2008.

10. Graziani G and Szabó C: Clinical perspectives of PARP inhibitors. Pharmacol Res 52: 109-118, 2005.

11. Szabó C: Pharmacological inhibition of poly(ADP-ribose) polymerase in cardiovascular disorders: Future directions. Curr Vasc Pharmacol 3: 301-303, 2005.

12. Boerma M, Wang J, Sridharan V,Herbert JM and Hauer-Jensen M: Pharmacological induction of transforming growth factor-betal in rat models enhances radiation injury in the intestine and the heart. PLoS One 8: e70479, 2013.

13. Santos LA, Ribeiro EL, Barbosa KP, Fragoso IT, Gomes FO, Donato MA, Silva BS, Silva AK, Rocha SW, França ME, et al: Diethylcarbamazine inhibits NF-kB activation in acute lung injury induced by carrageenan in mice. Int Immunopharmacol 23: 153-162, 2014

14. da Silva BS, Rodrigues GB, Rocha SW, Ribeiro EL, Gomes FO, E Silva AK and Peixoto CA: Inhibition of NF-kB activation by diethylcarbamazine prevents alcohol-induced liver injury in C57BL/6 mice. Tissue Cell 46: 363-371, 2014.

15. Lai D, Sun J, Li Y and He B: Usefulness of ventricular endocardial electric reconstruction from body surface potential maps to noninvasively localize ventricular ectopic activity in patients. Phys Med Biol 58: 3897-3909, 2013.

16. Menon SC, Cetta F, Dearani JA, Burkhart HA, Cabalka AK and Hagler DJ: Hybrid intraoperative pulmonary artery stent placement for congenital heart disease. Am J Cardiol 102: 1737-1741, 2008.

17. Ka SM, Kuoping Chao L, Lin JC, Chen ST, Li WT, Lin CN, Cheng JC, Jheng HL, Chen A and Hua KF: A low toxicity synthetic cinnamaldehyde derivative ameliorates renal inflammation in mice by inhibiting NLRP3 inflammasome and its related signaling pathways. Free Radic Biol Med 91: 10-24, 2016.

18. Mezzaroma E, Toldo S, Farkas D, Seropian IM, Van Tassell BW, Salloum FN, Kannan HR, Menna AC, Voelkel NF and Abbate A: The inflammasome promotes adverse cardiac remodeling following acute myocardial infarction in the mouse. Proc Natl Acad Sci USA 108: 19725-19730, 2011.

19. Mezzaroma E, Toldo S and Abbate A: Role of NLRP3 (cryopyrin) in acute myocardial infarction. Cardiovasc Res 99: 225-226, 2013.

20. Altaf A, Qu P, Zhao Y, Wang H, Lou D and Niu N: NLRP3 inflammasome in peripheral blood monocytes of acute coronary syndrome patients and its relationship with statins. Coron Artery Dis 26: 409-421, 2015.

21. Alestalo K, Miettinen JA, Vuolteenaho O, Huikuri $\mathrm{H}$ and Lehenkari P: Bone marrow mononuclear cell transplantation restores inflammatory balance of cytokines after ST segment elevation myocardial infarction. PLoS One 10: e0145094, 2015.

22. Ruparelia N, Digby JE, Jefferson A, Medway DJ, Neubauer S, Lygate CA and Choudhury RP: Myocardial infarction causes inflammation and leukocyte recruitment at remote sites in the myocardium and in the renal glomerulus. Inflamm Res 62: 515-525, 2013.

23. Zaitone SA and Abo-Gresha NM: Rosuvastatin promotes angiogenesis and reverses isoproterenol-induced acute myocardial infarction in rats: Role of iNOS and VEGF. Eur J Pharmacol 691: 134-142, 2012.

24. Das B and Sarkar C: Is preconditioning by oxytocin administration mediated by iNOS and/or mitochondrial K(ATP) channel activation in the in vivo anesthetized rabbit heart? Life Sci 90: 763-769, 2012.

25. Ørn S, Ueland T, Manhenke C, Sandanger $\varnothing$, Godang K, Yndestad A, Mollnes TE, Dickstein K and Aukrust P: Increased interleukin-1b levels are associated with left ventricular hypertrophy and remodelling following acute ST segment elevation myocardial infarction treated by primary percutaneous coronary intervention. J Intern Med 272: 267-276, 2012.

26. Li S, Fan Q, He S, Tang T, Liao Y and Xie J: MicroRNA-21 negatively regulates Treg cells through a TGF- $\beta 1 /$ Smad-independent pathway in patients with coronary heart disease. Cell Physiol Biochem 37: 866-878, 2015. 
27. Rocha SW, de França ME, Rodrigues GB, Barbosa KP Nunes AK, Pastor AF, Oliveira AG, Oliveira WH, Luna RL and Peixoto CA: Diethylcarbamazine reduces chronic inflammation and fibrosis in carbon tetrachloride- $\left(\mathrm{CCl}_{4}{ }^{-}\right)$induced liver injury in mice. Mediators Inflamm 2014: 696383, 2014.

28. Davies NM, Smith GD, Windmeijer F and Martin RM: COX-2 selective nonsteroidal anti-inflammatory drugs and risk of gastrointestinal tract complications and myocardial infarction: An instrumental variable analysis. Epidemiology 24: 352-362, 2013.

29. Varas-Lorenzo C, Castellsague J, Stang MR, Perez-Gutthann S, Aguado J and Rodriguez LA: The use of selective cyclooxygenase-2 inhibitors and the risk of acute myocardial infarction in Saskatchewan, Canada. Pharmacoepidemiol Drug Saf 18 1016-1025, 2009.
30. Metcalfe C, Wheeler BW, Gunnell D and Martin RM: International regulatory activity restricting COX-2 inhibitor use and deaths due to gastrointestinal haemorrhage and myocardial infarction. Pharmacoepidemiol Drug Saf 19: 778-785, 2010.

31. Ribeiro EL, Barbosa KP, Fragoso IT, Donato MA, Gomes FO, da Silva BS, Soares e Silva AK, Rocha SW, da Silva Junior VA and Peixoto CA: Diethylcarbamazine attenuates the development of carrageenan-induced lung injury in mice. Mediators Inflamm 2014: 105120, 2014

32. Dong F, Yang XJ, Jiang TB and Chen Y: Ischemia triggered ATP release through Pannexin-1 channel by myocardial cells activates sympathetic fibers. Microvasc Res 104: 32-37, 2016. 